\title{
Localization System for Mobile Robot Using Wireless Communication with IR Landmark
}

\author{
Byungkon Sohn, Jaeyeong Lee, Heesung Chae, and Wonpil Yu
}

\begin{abstract}
The localization of robots is a main problem to solve to make the robots navigate autonomously. This paper introduces an embedded indoor localization system for mobile robot. The system consists of a localization sensor that computes location of itself and infrared landmarks that are wirelessly controlled by the localization sensor. The localization sensor which is combined with an image sensor detects pixel positions of infrared sources from the landmark and by the pixel position the sensor figures out its position( $x, y)$ and heading angle. The coverage of a localization space is enlarged by dynamically combining robot odometer and IR landmark. The advantages of the developed system can be found on its position accuracy, robustness and easy expansion of localization space.
\end{abstract}

Index Terms—artificial landmark, localization, mobile robot, navigation

\section{INTRODUCTION}

Robot localization is a main requirement for autonomous navigation of mobile robot. In the field of mobile robotics, localization technology refers to a systematic approach to determine the current location of a mobile robot, namely, the 2-D position and heading angle of the mobile robot, by acquiring environmental information from robot sensors. The research for localization technology in the field of mobile robotics has been rigorously conducted so far; a good overview on the robot localization technology can be found in [1].

Over the decades to solve the localization problem, techniques using wireless communication, natural landmarks, and artificial landmarks etc. were used. The location sensing techniques using wireless communications are GPS (Global Positioning System) [2] and RF signal-based systems. On these systems a receiver takes signals from at least three transceivers to calculate its location. Triangulation techniques based on RSSI (Received Signal Strength Indicator), TOA (Time-Of-Arrival), AOA (Angle-Of-Arrival), and TDOA

Byungkon Sohn is with the Electronics and Telecommunications Research Institute, 161 Gajeongno, Yuseong-gu, Daejeon, 305-700, South Korea (phone: +82-42-860-5785; fax:+82-42-860-6790; e-mail: bksohn@etri.re.kr)

Jaeyeong Lee is with the Electronics and Telecommunications Research Institute, 161 Gajeongno, Yuseong-gu, Daejeon, 305-700, South Korea (e-mail: jylee@etri.re.kr)

Heesung Chae is with the Electronics and Telecommunications Research Institute, 161 Gajeongno, Yuseong-gu, Daejeon, 305-700, South Korea (e-mail: hschae@etri.re.kr)

Wonpil $\mathrm{Yu}$ is with the Electronics and Telecommunications Research Institute, 161 Gajeongno, Yuseong-gu, Daejeon, 305-700, South Korea (e-mail: ywp@etri.re.kr)
(Time-Difference-Of-Arrival) are used for localization. But localization techniques using RF signal-based communication have low accuracy on its localization result because of multi-path fading, interference, and non-LOS conditions. Though ultra wideband (UWB) system have attracted considerable attention to achieve accurate localization information, localization technique using UWB provides about $1 \mathrm{~m}$ position error for the moving object localization.[3][4]

Research about the localization using the natural landmarks has been developed from the field of computer vision. [5] Computer vision based localization has some good result but, it has yet some weaknesses; its computation complexity, inaccurate localization for navigation and instability in illumination changes.

The most ideal sensor for indoor localization should have the following attributes: high accuracy, stability, and robustness. For flexible motion control robot should update its localization information in real-time. The localization space should be easily expandable to be used in home or office environment. The localization sensor should not be affected by the sunlight or other illumination changes occurred in indoor environment. Among the localization sensors developed until now, there are very few sensors that meet requirements mentioned above.

To solve this problem, we used wireless sensor network based on artificial landmarks. Recently, there have been remarkable developments in the wireless communication field. Through the growth of wireless communication, smart home applications using wireless sensor network have been actively researched [6][7][8]. Using sensor network for robot localization is a promising approach because the intelligent buildings will be actualized in the future life.

This paper proposes a localization system for mobile robot using wireless communication with artificial landmark. The localization system is composed of a localization image sensor and IR (infrared) artificial landmarks. The image sensor installed on the robot captures the image of IR landmarks which are attached on the ceiling. The sensor calculates its position relative to the landmark position when the sensor finds two IR landmarks.

The proposed localization system provides robot with accurate and stable location information. The system can update location in real-time while the robot is moving. The space covered by the system can be easily expandable, because localization sensor in the system communicates with sensors deployed on indoor environment. Just by installing more sensors in the space, the space for robot localization can be 
expanded.

\section{ARCHITECTURE OF LOCALIZATION SYSTEM}

The proposed localization system consists of localization sensor and IR landmarks. The IR landmarks are attached on the ceiling of a space and the localization sensor is mounted on the top of a mobile robot as shown in Fig.1. The sensor is a camera module having an infrared band-pass filter. It is oriented to look upward so that its optical axis is perpendicular to the ground. In order to obtain maximal field of view, a wide-angle camera lens is utilized.

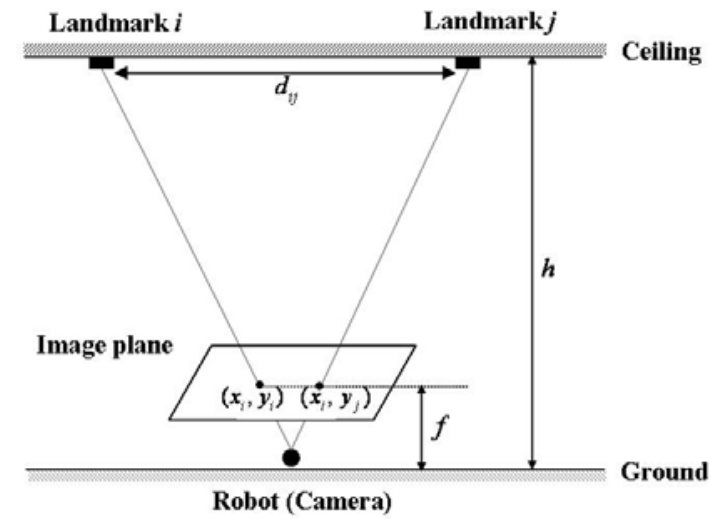

Fig. 1. Sensor configuration of the proposed location sensing system.

The localization sensor (Fig.2.(a)) has an embedded processor, an image sensor, a RF (radio frequency) chip for wireless communication, and a SDRAM memory. The embedded processor has software drivers to control the image sensor, the RF chip, a UART(universal asynchronous receiver/transmitter) and the SDRAM. It also has localization algorithm for detecting and tracking IR landmarks: the sensor computes its location in real-time by tracking the IR sources from the landmarks. Image sensor has its own registers to adjust image resolution, image color depth, and camera exposure. The sensor sends commands to IR landmark via RF chip (2.4GHz frequency) to control the wireless IR landmarks. There are three commands that the sensor sends to the landmarks: 'turn on' command, 'turn off' command, and request command for the remaining battery level of the landmarks. The SDRAM stores parameter data and program sources that are used during computing the location. The sensor calculates its location after recognizing the two landmark's position and it transfers its location data to robot via serial communication. The sensor has dimension of 37x37x42.6(mm) and due to the small size as localization sensor, the sensor can be easily installed on any type of robots. The IR landmark (Fig. 2. (b)) is composed of a microprocessor, a RF chip, and an IR LED.

The landmark uses two AA size batteries as its power. The microprocessor on landmark controls RF communication to receive messages from the sensor to turn on and off the IR LED. In order to control IR LEDs of the landmarks independently, a unique landmark ID is assigned to each IR landmark module.

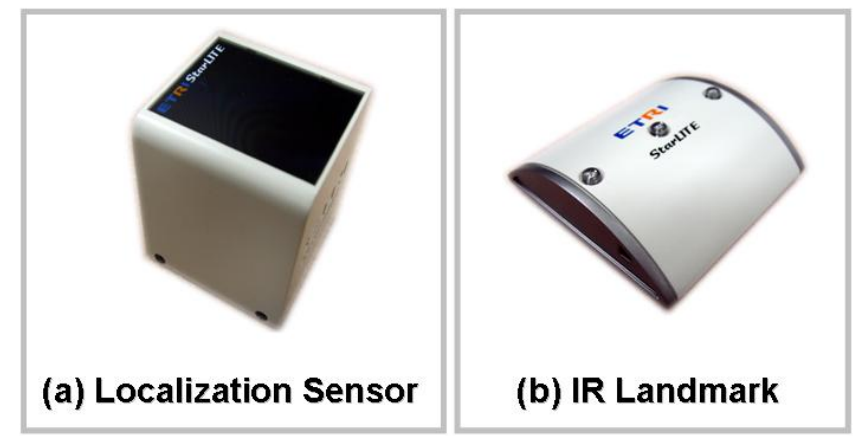

Fig. 2. (a) Localization Sensor, (b) IR Landmark

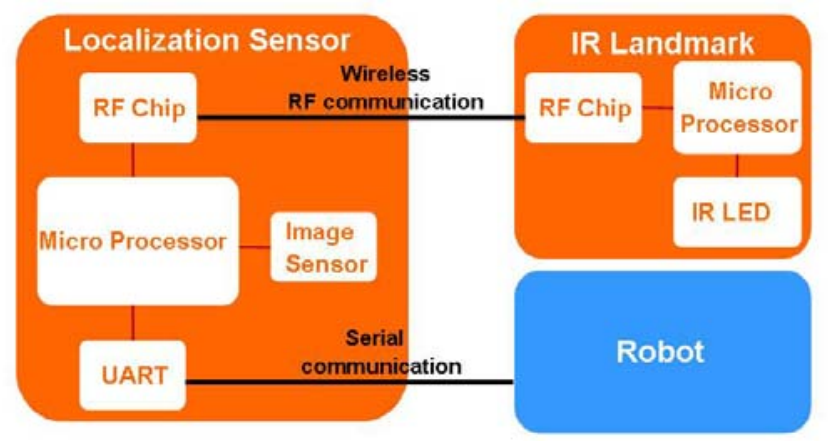

Fig. 3. Architecture of localization system.

\section{LOCALIZATION}

The location information of a robot can be obtained when at least two infrared LEDs are detected within the field of view of the camera. The localization is performed in two steps. In the first step, the image coordinates of the IR LEDs, if any, are computed and landmark IDs are identified. In the second step 2-D location and heading angle of a robot are computed from the image coordinates and the robot's position in image coordinate is transformed to the world coordinate by referencing world coordinate of the landmarks.

\section{A. Detection and Identification of IR Landmarks}

The IR band-pass filter attached on the localization sensor transmits only IR band by filtering out visible band. Fig.4 demonstrates the effect of infrared band-pass filtering. Fig.4(a) was captured by the camera sensor without filter and the Fig.4(b) was captured by sensor with filter on it. The IR LEDs are well discriminated as white spots in the image Fig.4(b). As well known in vision community, it is very difficult to robustly locate particular patterns from images in varying illumination condition. Our IR band-pass filtering solution makes the detection problem a simple thresholding problem, and more importantly, it enables robust detection of target landmarks at any time regardless of day and night.

On the stage of computing initial robot position, to recognize the ID of the detected LED spot, only one infrared LED is turned on and the others turned off before capturing the image. If any spot is detected from the image, it is identified as being originated from the landmark which was set to be turned on. This detection and identification procedure is iterated for each landmark module until two landmark spots are detected. 


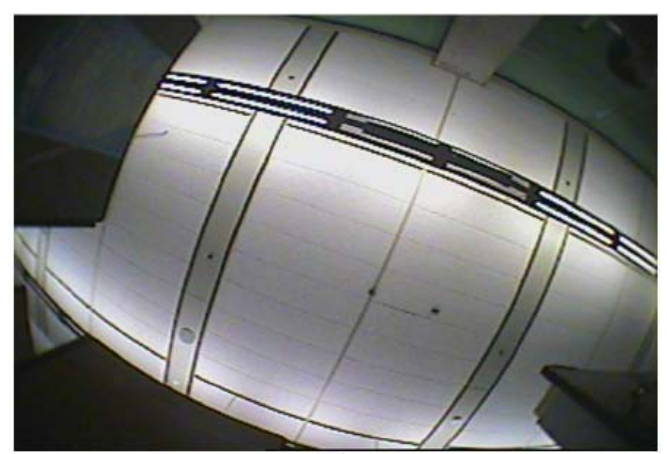

(a)

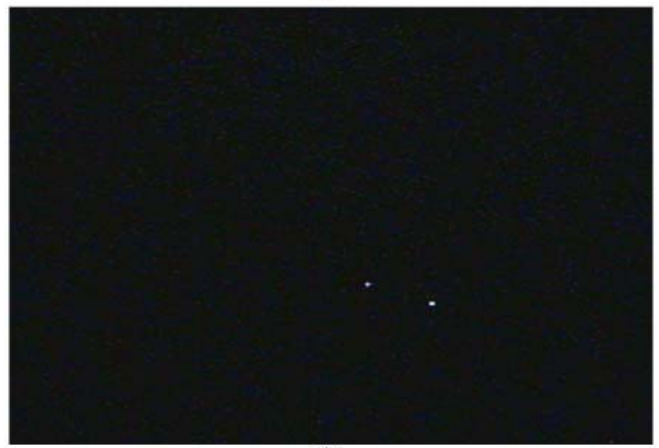

(b)

Fig. 4. Two sample images of a pair of infrared landmarks. (a) Image captured by the sensor without filter. (b) Image captured by the same sensor with an infrared band-pass filter.

Camera calibration is a necessary step in this localization sensing system for using a wide-angel lens. However, we had used the established calibration methods [9][10][11]. Especially, the main initialization for the camera calibration phase has been partially inspired from the Zhengyou Zhang' algorithm [12].

\section{B. Computation of 2-D Position and Heading Angle}

For the description of robot localization procedure, we use two coordinate systems: world coordinate system and image coordinate system. The world coordinate system represents the real position in the localization space and the image coordinate system refers to the pixel coordinate in the image. Fig. 5 shows the relationship between world coordinate and image coordinate.

Let $L_{i}$ and $L_{j}$ be the two LEDs located in the image in the previous step. Let $\left(x_{i}, y_{i}\right)$ and $\left(x_{j}, y_{j}\right)$ be image coordinates of the two LEDs and let $\left(X_{i}, Y_{i}\right)$ and $\left(X_{j}, Y_{j}\right)$ be the corresponding world coordinates. We assume that the world coordinate of each landmark is already known. We can set the image coordinate of the robot to be the center of the image, denoted by $\left(c_{x}, c_{y}\right)$, by assuming that the optical center of the camera is coincident with the rotating axis of the robot. The world coordinate of the robot's position, denoted by $\left(r_{x}, r_{y}\right)$, is computed by applying the transformation that transforms from the image coordinate of $L_{i}$ and $L_{j}$ to the corresponding world coordinates to the image center, $\left(c_{x}, c_{y}\right)$. Let $\theta$ be the heading angle of the robot on the basis of $y$-axis of the world coordinate system. The location of the robot is then given by

$$
\begin{aligned}
& \cos \theta=\frac{\left(x_{j}-x_{i}\right)\left(X_{j}-X_{i}\right)+\left(y_{j}-y_{i}\right)\left(Y_{j}-Y_{i}\right)}{d D} \\
& \sin \theta=\frac{\left(x_{j}-x_{i}\right)\left(Y_{j}-Y_{i}\right)-\left(y_{j}-y_{i}\right)\left(X_{j}-X_{i}\right)}{d D}, \\
& {\left[\begin{array}{l}
r_{x} \\
r_{y}
\end{array}\right]=s\left[\begin{array}{cc}
\cos \theta & -\sin \theta \\
\sin \theta & \cos \theta
\end{array}\right]\left(\left[\begin{array}{l}
c_{x} \\
c_{y}
\end{array}\right]-\left[\begin{array}{c}
x_{i} \\
y_{i}
\end{array}\right]\right)+\left[\begin{array}{c}
X_{i} \\
Y_{i}
\end{array}\right]}
\end{aligned}
$$

where $D$ is the distance from $L_{i}$ to $L_{j}$ in world coordinate system, $d$ is the distance from $L_{i}$ to $L_{j}$ in image coordinate system, $s$ denotes a scale factor between two coordinate system.

$$
s=\frac{D}{d}=\frac{\sqrt{\left(X_{i}-X_{j}\right)^{2}+\left(Y_{i}-Y_{j}\right)^{2}}}{\sqrt{\left(x_{i}-x_{j}\right)^{2}+\left(y_{i}-y_{j}\right)^{2}}}
$$

In the next section, we describe the method of identifying the detected landmarks in real-time and extending the coverage of a localization network to arbitrary large space.

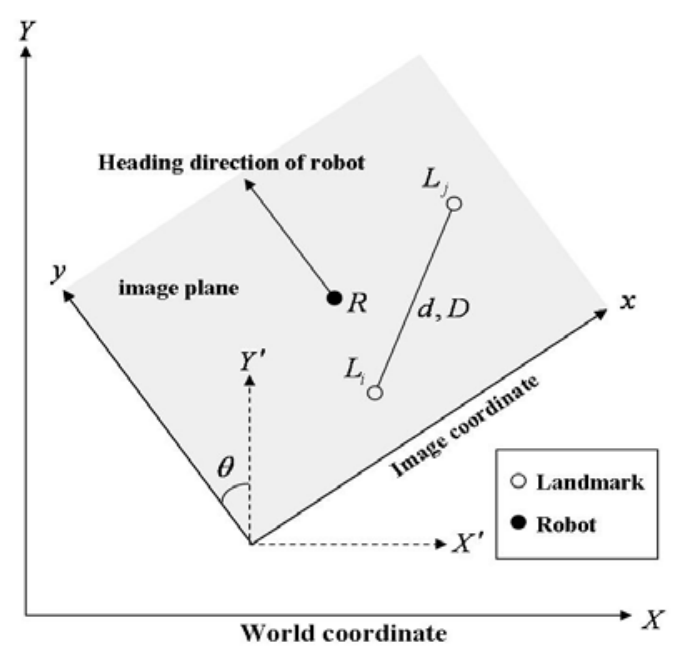

Fig. 5. Two coordinate systems used in robot localization.

\section{REAL-TIME LANDMARK IDENTIFICATION AND LOCALIZATION}

The location information of a robot can be obtained when at least two IR landmark are detected within the field of view of the camera. Therefore, a lot of landmarks are required to cover the whole area of a large indoor environment. There are two possibilities of disposing the landmarks in the space. First, as shown in Fig. 6(a), we can arrange the landmarks such that at least two landmarks are seen by the camera at any location within the localization space. In this case, a mobile robot can be self-localized by using only these landmarks, but the cost would increase unfeasibly for a large environment. Second way is to deploy IR landmarks sparsely in the space, mainly at important places such as corners and entrance of narrow passage as illustrated in Fig. 6(b). In this case, we need another solution to locate a robot in places where IR landmarks are not 
detected by the camera sensor. In our system, we adopts dead-reckoning to solve this problem, which is a relative localization method based on the odometer from the wheel velocity of a mobile robot. The Dead-reckoning has the problem of accumulation of errors over time but it is fairly accurate for a short trip [13].

The proposed localization system works in two main steps of initial localization and real-time update of the location. Fig. 7 shows the overall procedure of the proposed localization system. The initial localization is a step of firstly estimating the location of a robot under having no information on its location. After initial localization, the location of a robot is updated in real-time by combining landmark-based localization and dead-reckoning dynamically.

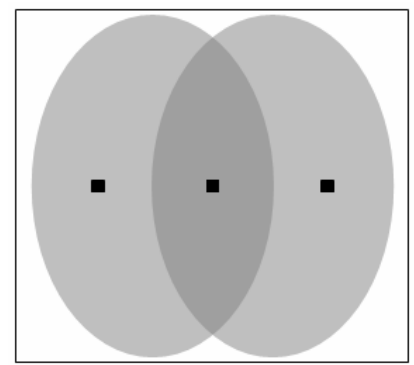

(a)

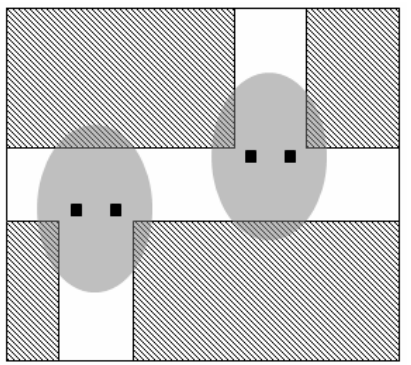

(b)
Fig. 6. Two kinds of disposition of landmarks for building a localization network. (a) Continuous disposition. (b) Sparse disposition.

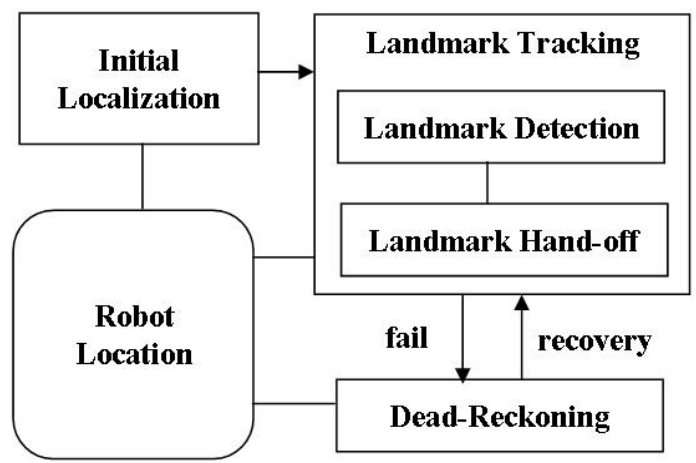

Fig. 7. Overall operating procedure of the proposed localization system.

Initially, the location of a robot is computed by tracking the landmarks which is detected in the initial localization step (mentioned in section III-A). The landmarks being tracked will eventually disappear from the view of the camera as the robot moves. In this case, Hand-off is occurred if any landmark is newly detected, or the localization mode is switched to dead-reckoning otherwise.

\section{A. Landmark Tracking and Hand-off Processing}

The location of a robot is computed and updated for every frame of camera image. Since the computational cost is trivial, the update rate of the location is about $30 \mathrm{~Hz}$.

Let us first suppose that we have determined the location of a robot, denoted by $\left(r_{x}^{t-1}, r_{y}{ }^{t-1} ; \theta^{t-1}\right)$, by detecting and identifying two landmarks, denoted by $L_{i}$ and $L_{j}$, from the image captured at time $t-1$. Let $\left(x_{i}^{t-1}, y_{i}^{t-1}\right)$ and $\left(x_{j}^{t-1}, y_{j}^{t-1}\right)$ be the detected image coordinates of the landmarks and $\left(X_{i}, Y_{i}\right)$ and $\left(X_{j}, Y_{j}\right)$ be the corresponding world coordinates respectively. Initially, these IR landmarks are set to the ones detected in the initial localization step.

1) Landmark Tracking: We capture a new image and detect LED spots in the image at time $t$. Instead of detecting LEDs in the whole image, the LEDs are searched within only searching areas as shown in Fig. 8(a). The size of searching area is determined based on the maximal velocity of the robot, frame rate of the camera, scale factor $s$, and the radial distance of the LED from the center of image.

Let $S_{i}$ be the searching area centered at $\left(x_{i}^{t-1}, y_{i}^{t-1}\right)$ and $S_{j}$ be the searching area centered at $\left(x_{j}^{t-1}, y_{j}^{t-1}\right)$. A simple nearest neighbor rule is to identify the closest LED spot detected in $S_{i}$ as $L_{i}$ and the closest LED spot detected in $S_{j}$ as $L_{j}$. However, if the searching areas are overlapped due to high velocity of a robot, both $L_{i}$ and $L_{j}$ could be mapped to the same LED spot as illustrated in Fig. 8(b). To solve this problem, we use a pair-wise nearest neighbor rule of identifying each LED spot such that the largest displacement is minimized. It can be considered as a strategy of minimizing the highest cost.

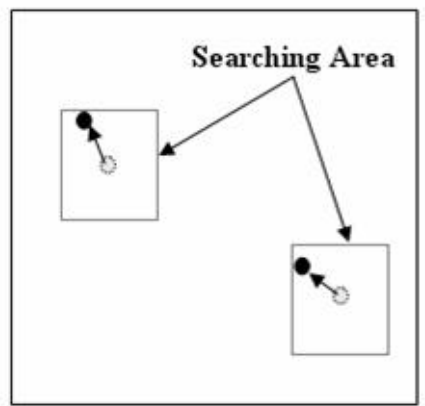

(a)

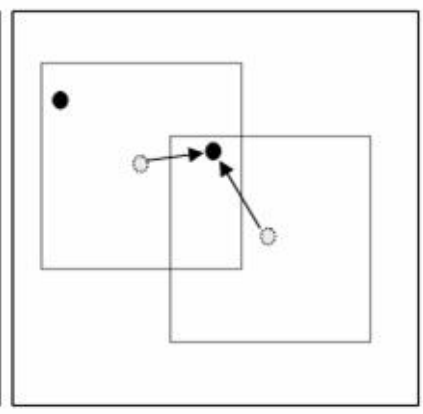

(b)
Fig. 8. (a) Searching area for landmark tracking. (b) An example of incorrect tracking.

If two landmarks are successfully detected in the searching areas, we can compute and update the location of the robot since they are already identified. On the other hand, if it fails to detect both landmarks, the detection is performed for the whole image in order to find the landmarks that come to newly enter within the view of the camera as the robot moves. If any new landmark is detected out of the searching areas, then hand-off is occurred. In other case, the mode of localization is switched to dead-reckoning.

2) Hand-off Processing: Let us suppose that a landmark $L$ is detected at $(x, y)$ in the current image. In order to identify landmark $L$, image coordinate of each landmark is inversely estimated from the latest location of the robot, $\left(r_{x}{ }^{t-1}, r_{y}{ }^{t-1} ; \theta^{t-1}\right)$. Let $L_{1}, L_{2}, \ldots, L_{n}$ be the landmarks installed in the space and $\left(X_{1}\right.$, $\left.Y_{1}\right),\left(X_{2}, Y_{2}\right), \ldots,\left(X_{n}, Y_{n}\right)$ be the corresponding world coordinates of the landmarks. The estimated image coordinate of the landmark $L_{k}$, denoted by $\left(x_{k}^{\prime}, y_{k}{ }^{\prime}\right)$, is computed by 


$$
\left[\begin{array}{l}
x_{k}{ }^{\prime} \\
y_{k}{ }^{\prime}
\end{array}\right]=\left[\begin{array}{l}
c_{x} \\
C_{y}
\end{array}\right]-\frac{1}{s}\left[\begin{array}{cc}
\cos \theta^{t-1} & \sin \theta^{t-1} \\
-\sin \theta^{t-1} & \cos \theta^{t-1}
\end{array}\right]\left(\left[\begin{array}{l}
r_{x}^{t-1} \\
r_{y}^{t-1}
\end{array}\right]-\left[\begin{array}{c}
X_{k} \\
Y_{k}
\end{array}\right]\right)
$$

for $k=1,2, \ldots, n$, where $s$ is the scale factor computed in the initial localization step.

The newly detected landmark $L$ is then identified as the landmark that has the closest estimated coordinate with $(x, y)$ :

$$
L=L_{k} \mid \arg _{k} \min \sqrt{\left(x_{k}{ }^{\prime}-x\right)^{2}+\left(y_{k}{ }^{\prime}-y\right)^{2}} .
$$

\section{B. Combining with Dead-reckoning}

So far, we have described landmark-based localization of a robot, which works only when at least two IR landmarks are visible from the camera sensor. In case that a robot strays out of the localization coverage of the landmarks or landmarks having been tracked are temporarily occluded, the mode of the localization system is switched to dead-reckoning for the sake of seamless provision of the location information. In the mode of dead-reckoning, the location is relatively updated based on the odometer from the wheel velocity of the mobile robot.

Let $\left(r_{x}{ }^{-1}, r_{y}{ }^{t-1} ; \theta^{t-1}\right)$ be the location of the robot at time $t-1, v_{l}$ and $v_{r}$ be the velocities of the left and right wheels measured at time $t, W$ be the length of baseline between two wheels, and $\Delta t$ be the time interval from time $t-1$ to time $t$. Then, location of the robot at time $t,\left(r_{x}{ }^{t}, r_{y}{ }^{t} ; \theta^{t}\right)$, is given by

$$
\begin{aligned}
& r_{x}^{t}=r_{x}^{t-1}+\Delta T \cos \left(\theta^{t-1}+\frac{\Delta R}{2}\right) \\
& r_{y}^{t}=r_{y}^{t-1}+\Delta T \sin \left(\theta^{t-1}+\frac{\Delta R}{2}\right) \\
& \theta^{t}=\theta^{t-1}+\Delta R
\end{aligned}
$$

where $\Delta T$ is the translational term and $\Delta \theta$ is the rotational term, which are given by

$$
\begin{aligned}
& \Delta T=\frac{v_{l}+v_{r}}{2} \Delta t \\
& \Delta R=\frac{v_{r}-v_{l}}{W} \Delta t
\end{aligned} .
$$

The location information of the robot is thus updated iteratively by (5) until any two landmarks are visible in the camera. On each iteration, an image is captured and tested for the detection of infrared landmarks. If two landmarks are detected in the image, the mode of the localization system is converted to landmark-based localization by using the method previously described in the hand-off process. Firstly, image coordinates of all the landmarks in the space are inversely estimated from the latest location of the robot, which is computed using the dead-reckoning. Detected landmarks are then identified using the nearest neighborhood rule. Once identified, subsequent localization is performed based on landmark tracking.

\section{EXPERIMENTAL RESULT}

For the evaluation of localization performance of the proposed localization system, we have conducted an experiment by changing the position and heading angle of a mobile robot. The experiment was performed in an indoor environment where three IR landmarks are attached on the ceiling. Each distance among IR landmarks is $120 \mathrm{~cm}$. The location information was computed for every grid points which are regularly spaced by the distance of $60 \mathrm{~cm}$. Fig. 9 shows the localization results using the proposed localization system. The estimated positions are represented by circles and true positions with asterisk. The mean position error is $4.1 \mathrm{~cm}$ with standard deviation of $2.9 \mathrm{~cm}$.

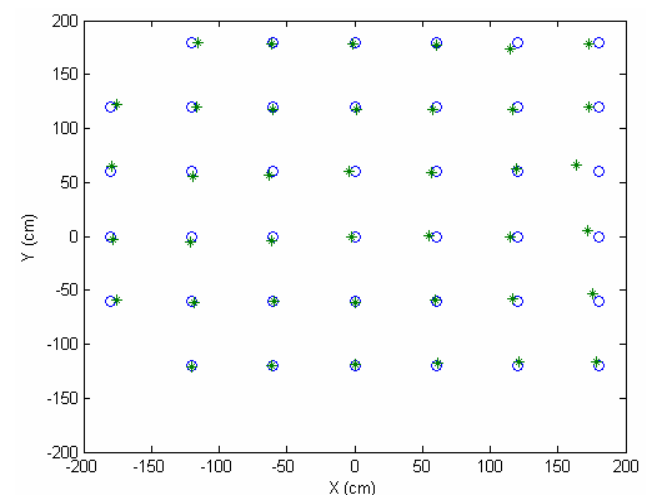

Fig. 9. Localization results with the proposed localization system.

We have the maximum position error of $17.1 \mathrm{~cm}$ at $(180,60)$ because of uneven ground condition. The error of location is minimized on the even ground. The error caused by uneven surface can be easily detected and corrected by incorporating odometer readings.

For measuring heading angle error, we turn the localization sensor by $10^{\circ}$ from $0^{\circ}$ to $360^{\circ}$. The Fig. 10 shows the result of heading angle error. The mean angle error was 0.26 degree and its deviation was 0.21 degree.

Position jitter was also measured to be less than $0.5 \mathrm{~cm}$ and $0.3^{\circ}$, which well explains the reliability of the proposed system.

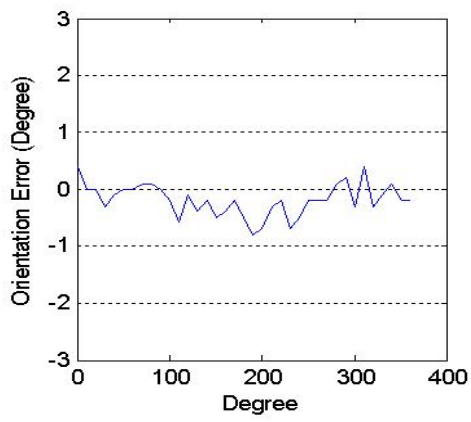

Fig. 10. Heading angle error.

The developed localization system and its extension to localization network were implemented to build a ubiquitous robotic space (URS) [14]. A prototype URS was implemented 
in the ground floor of our building, the area of which was measured to be $22.8 \mathrm{~m} \times 21.6 \mathrm{~m}$. A sensor network based on ZigBee protocol was also installed in the same space to gather environmental data along with the localization information to infer contextual information.

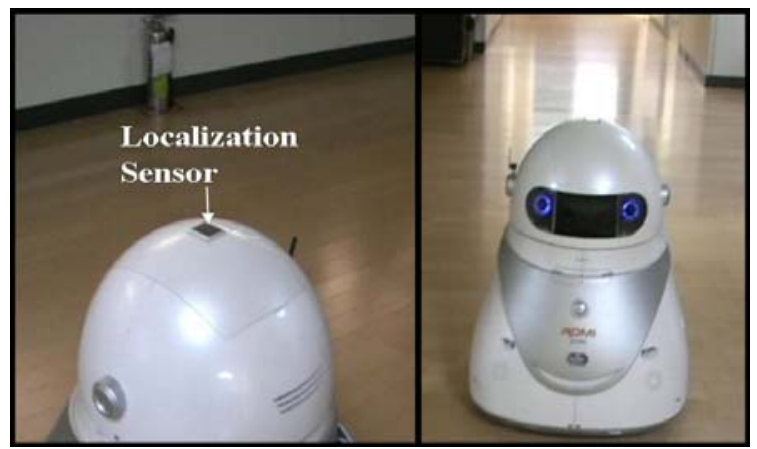

(a)

(b)

Fig. 11. (a) Localization sensor mounted on the robot's head. (b) Test robot for URS : ROMI

Fig. 12 shows the metric map of the space. Thick blue lines represent the trajectory of the robot (ROMI) along which the robot carries out a routine monitoring task. Small dots represent the positions of the developed localization landmarks and the landmark pairs can be arranged in any direction. The arrangement of landmarks does not affect the localization performance if the distance between landmark pair maintains a proper range: normally $30 \mathrm{~cm} \sim 150 \mathrm{~cm}$ on $2.5 \mathrm{~m}$ ceiling height. The large green dots represent the positions of the ZigBee sensor nodes. Irregular situation is detected from the contextual information processing in the semantic space, which subsequently issues a new navigation task to the robot to visit designated spot. The whole system can be monitored on a remote PC or PDAs; irregular situation is promptly reported to the user through CDMA communications network. By integrating all the elements above, a robotic security application was experimentally implemented and it was found that the proposed localization system provides information with acceptable performance for carrying out robotic tasks according to contextual information in the space.

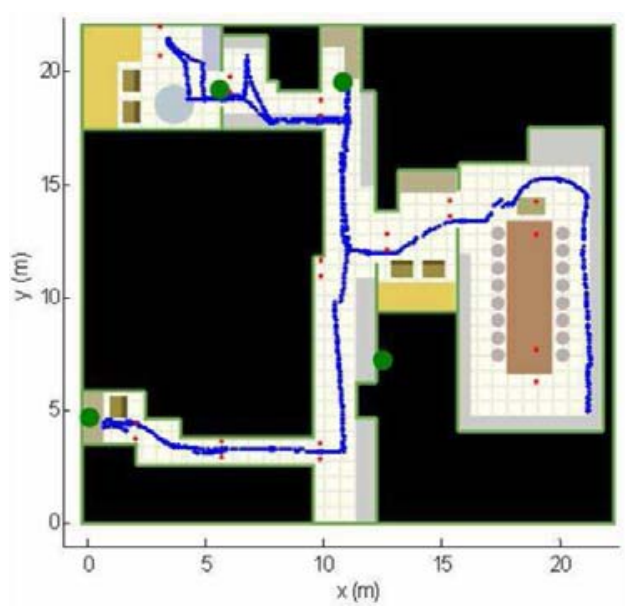

Fig. 12. Metric map of the developed ubiquitous robotic space.

\section{CONCLUSION}

In this paper, we have proposed an embedded robot localization system which can be applied to develop a ubiquitous robotic space for a large indoor environment where a robot or human can have information assistance from the space and a specific robotic task can be carried out efficiently. Experimental result confirmed the robustness of localization data and its accuracy. By implementing a prototype ubiquitous robotic space(URS), we could be assured that the proposed localization network well satisfied the design constraints imposed for developing a realistic space technology. One of our future works includes automatically locating the landmarks by using modern mapping, or SLAM techniques.

\section{ACKNOWLEDGEMENT}

This work was supported in part by Korea MIC and IITA through IT leading R\&D Support Program (2005-S-092-03, USN-based Ubiquitous Robotic Space Technology Development).

\section{REFERENCES}

[1] J. Borenstein, H. R. Everett, and L. Feng, "Where am I? Sensors and Methods for Mobile Robot Positioning,” Technical Report, Univ. of Michigan, APR. 1996.

[2] T. S. Rappaport, J. H. Reed, and B. D. Woerner, "Position Location Using Wireless Communications on Highways of the Future," IEEE Communications Magazine, pp. 33-41, OCT. 1996.

[3] J. Y. Lee and R. A. Scholtz, "Ranging in a Dense Multipath Environment Using an UWB Radio Link,” IEEE J. Selected Areas in Communications, vol. 20, no. 9, DEC. 2002.

[4] The Ubisense Smart Space Platform, http://www.ubisense.net.

[5] D.C.K. Yuen and B.A.MacDonald, "Vision-based localization algorithm based on landmark matching, triangulation, reconstruction, and comparison,” IEEE Trans. Robotics, vol. 21, no. 2, pp. 217-226, APR. 2005.

[6] J.H.Lee, K.Morioka, N.Ando, H.Hashimoto, “Cooperation of Distributed Intelligent Sensors in Intelligent Environmet,” IEEE/ASME Trans. Mechatronics, vol. 9, no. 3, SEP. 2004.

[7] N.Y.Chong, H.Hongu, K.Ohba, S.Hirai, and K.Tanie, "A Distributed Knowledge Network for Real World Robot Applications,” IEEE Int. Conf. Intell. Robots and Systems, pp. 187-192, 2004.

[8] D.Haehnet, W.Burgard, D.Fox, K.P.Fishkin, M.Philipose, "Mapping and Localization with RFID Technology," IEEE Int. Conf. Robotics and Automation, pp.1015-1020, 2004.

[9] R.Y.Tsai,, "A versatile camera calibration technique for high accuracy 3D machine vision metrology using off-the-shelf TV cameras and lenses,” IEEE J. Robotics Automat., pages 323-244, Vol. RA-3, No.41987.

[10] T.A. Clarke and J.G. Fryer, "The Development of Camera Calibration Methods and Models," Photogrammetric Record, 16(91): 51-66, April 1998.

[11] Sturm and Maybank, "On Plane-Based Camera Calibration: A General Algorithm, Singularities, Applications,” CVPR99

[12] Zhang, "Flexible Camera Calibration by Viewing a Plane from Unknown Orientations," ICCV99

[13] B. Yamauchi, "Mobile robot localization in dynamic environments using dead reckoning and evidence grids," in Proc. 1996 IEEE Conf. Robotics and Automation, pp. 1401-1406.

[14] Young-Guk Ha, Joo-Chan Sohn, Young-Jo Cho, and Hyunsoo Yoon, "Towards Ubiquitous Robotic Companion: Design and Implementation of Ubiquitous Robotic Service Framework”, ETRI Journal, vol.27, no.6, Dec. 2005, pp.666-676 